Bull. Mater. Sci., Vol. 17, No. 6, November 1994, pp. 841-848. (C) Printed in India.

\title{
Creation of nanostructures on nickel thin films by STM
}

\author{
V SRINIVAS, M V H RAO, B K MATHUR and K L CHOPRA \\ Department of Physics and Meteorology, Indian Institute of Technology, Kharagpur 721 302, India
}

\begin{abstract}
A scanning tunneling microscope (STM) has been used to create nanostructures on nickel thin films. A systematic procedure for the creation of such structures has been developed. Various possible mechanisms involved in such creations have been discussed. Based on our observations, a field-induced suction of the plastically deformed surface has been proposed.
\end{abstract}

Keywords. Nickel; thin film, nanostructures; STM.

\section{Introduction}

One of the subjects of great current interest in scanning tunneling microscopy (STM) is its application in the manipulation of atoms and molecules on solid surfaces (Becker et al 1987; Rohrer 1993) and in creating lithographic structures on a nanoscale. The possibility of creation of structures such as quantum wires and quantum dots of highly specific dimensions at specified locations on a nanometre scale has opened up an exciting frontier in surface science and technology. Various surface modification techniques using STM are described as nanomachining (Virtanen et al 1991), nanoindentation (Schmmel et al 1992), nanoetching (Saulys et al 1991), nanostructuring using high field (Snow et al 1993), high current (Hartmann et al 1991), and pulse (Schmmel et al 1991), and nanolithography (McCord and Pease 1988). McCord and Pease $(1986,1987)$ have written 200-nm-wide lines on thin resist films using STM. Staufer et al $(1987,1988)$ created small hillocks of $\sim 35$ $\mathrm{nm}$ diameter by locally melting a glassy substrate. It has been shown by Schneir et al (1988) that 5-nm-wide lines can be written if the substrate is first covered by a fluorocarbon grease.

Fabrication of nanostructures on surfaces using STM can be attained either by tip-sample direct indentation, or by interaction at a distance between the two. In the former method, modification of the solid surface is usually achieved by locally destroying the surface, i.e. mechanically scratching the surface with the tip (Schneir et al 1988). In this process the tip loses its sharpness, thereby making it ineffective for subsequent topographic application. The other technique, which is referred to as tip bias pulsing method, is essentially a nondestructive method. In this technique, the main control parameters for 'writing pulse' are the sign, magnitude, duration and number of voltage pulses applied between the tip and the sample. Using this method one can create either valleys ( $\mathrm{Li}$ et al 1989) or hillocks (Mamin et al 1990). However, it has been observed that the shape of the created structure depends not only on the tip shape but also on the nature of the sample surface. In bias pulsing technique, the STM is operated in field emission mode for a very limited time. Recently there have been a series of investigations on gold as it is known to have a lower threshold for field evaporation compared to refractive metals. It 
has been pointed out that tip-sample direct interaction is also possible in the bias pulse technique (Pascual et al 1993). These coupled interactions may lead to structures completely different from what we expect. However, the real physical mechanism underlying this phenomenon is not well understood.

In the present investigation we have attempted to develop a procedure to use bias pulsing technique to create and modify nanometric structures on nickel thin films. We also discuss the possible physical mechanism underlying this phenomenon.

\section{Experimental}

Thin films of nickel of various thicknesses on different substrates were deposited using electron beam evaporation technique under high-vacuum conditions ( $10^{-7}$ torr). The samples were subsequently transferred to STM chamber, where these experiments were carried out in air. Electrochemically etched sharp tungsten tips (Rao and Mathur 1993) were used for topographic scan with $0.25 \mathrm{~V}$ bias voltage and 200 pA tunneling current in constant current mode. The bias pulsing method used in the present work for creation of nanostructures was done in the constant height configuration. This procedure has been followed in order to avoid tip-sample direct interaction. A digital storage oscilloscope was used to record pulse height and width precisely.

Prior to the surface modification, smooth areas were selected by scanning large areas at different locations on the sample surface. The average surface roughness was found to be $15-25 \AA$. These scans were also taken in order to make sure no structures were present prior to the surface modification. After the topographic scan with constant current configuration the tip was moved to a particular location where the surface modification was desired. Then we switched over to the constant height mode and subsequently the voltage pulse was directly added to the tunneling voltage.

The positioning of the tip, the shape of the tip and the shape of the pulse are important parameters governing nanostructuring. In our STM, the sample is mounted on a piezo tube and the position of the sample below the tip can be adjusted by controlling the voltage applied to the offset piezo. This facility permits us to shift the sample in a precisely controlled manner so as to position the tip at the required location on the surface.

The shape of the nanostructures is not an exact replica of the tip shape but is greatly influenced by it. Trials with various grounded tips produced different structures. As an example, the triple structures seen in figure 1 are most probably due to a tip having three pointed corners at different heights. In the imaging mode, the pointed corner closest to the surface is the main contributor to the tunneling current. But in the high-field mode, the three points of the tip generate different fields on the surface, thereby creating three hillocks of different sizes. This has been checked at three different locations and we see that hillock structures are similar. In order to produce small symmetrical structures, it is desirable to use a tip which is pretested on standard sample for atomic resolution.

The voltage amplitude and width of the pulse necessary to produce nanostructures depend on the material of the surface. Earlier workers have reported voltage pulse widths in the range of a few microseconds to a few milliseconds for different 


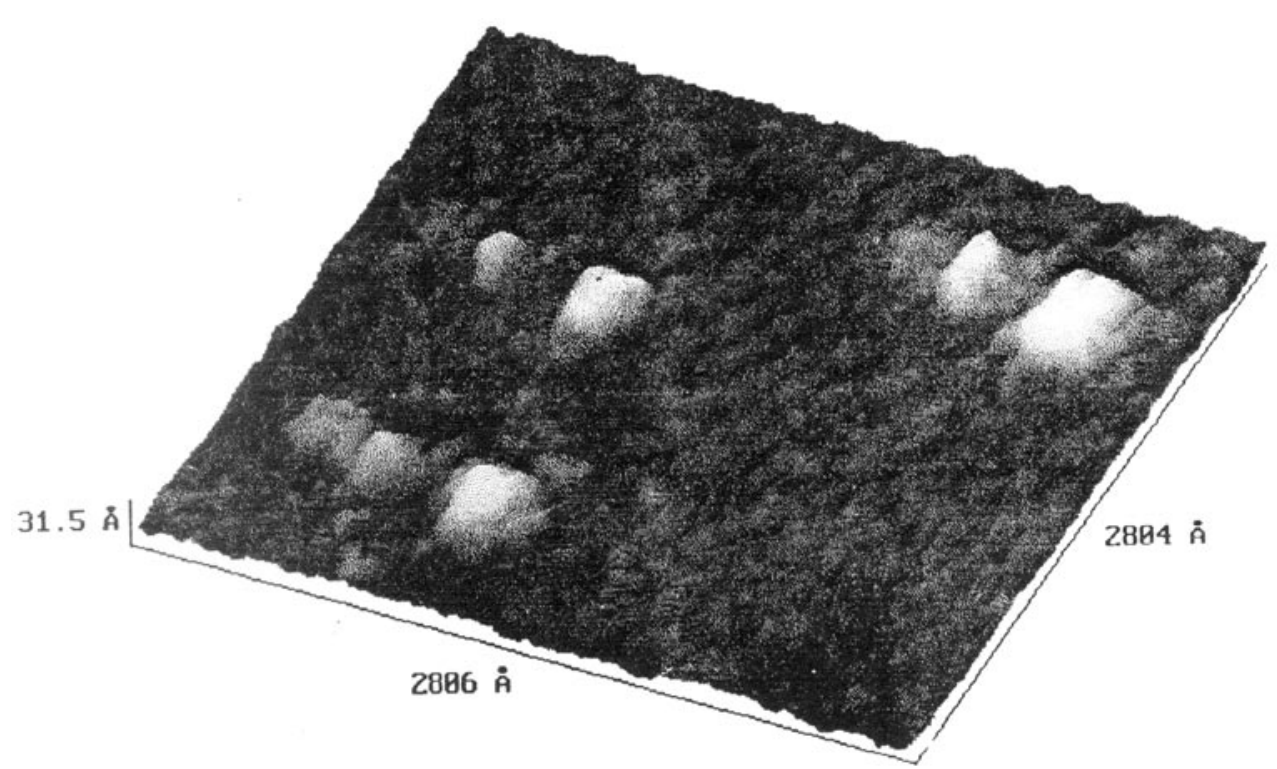

Figure 1. Nanostructures created by a triple-pointed tip at three different locations.

materials. Our trials show that for production of stable plastic deformation on the surface of nickel film on mica, a $100-\mathrm{ms}$-wide pulse is sufficient. It is found that the structure could be created only with a certain minimum pulse height for a specified tip-sample distance. We observed that the writing probability goes from 0 to $100 \%$ within a fraction of a volt and this voltage is determined by repeating the experiment several times. The threshold voltage for nickel was found to be $2.5 \mathrm{~V}$ when the tunneling conditions just before writing were $V=0.25 \mathrm{~V}$ and $I=$ $200 \mathrm{pA}$, below which there is no surface modification. At $2.5 \mathrm{~V}$, formation of hillocks commences of height $\sim 50 \AA$ and diameter $\sim 200 \AA$. As the voltage increases, the overall size increases but the shape remains more or less the same. The inner two hillocks in figure 2 , were formed with a bias pulse of $3.0 \mathrm{~V}$ whereas the outer ones were created by a $4.0 \mathrm{~V}$ pulse. It is seen that the hillocks created by $4.0 \mathrm{~V}$ pulse are larger than those created by $3.0 \mathrm{~V}$ pulse but the overall shape remains more or less the same. A similar behaviour as seen in figures 1 and 2 has been observed in a large number of trials. The observations suggest that the shape of the hillock is governed by the shape of the tip but the overall size is determined by the effective field strength on the surface. A pulse greater than $4.5 \mathrm{~V}$ produces drastic changes in the surface topography.

A single-parameter quantification of surface modification is desirable. This involves measurement of physical dimensions of the nanostructures. Although it is possible to measure these dimensions using image processing techniques this only generates a large amount of data. Fractal characterization in terms of scaling exponent is difficult to recommend because the surface can no longer be regarded as self-affine due to area-specific modifications. In order to standardize the nano-structuring procedure, it is necessary to use a parameter which defines the extent of modification. 
As the structures produced during our investigations are in the shape of hillocks, a rough estimate of their volume is used in our investigations to characterize the nanostructures.

As the bias pulse amplitude increases, the volume of the nanostructures also increases. Figure 3 is one such plot characterizing the surface modification with

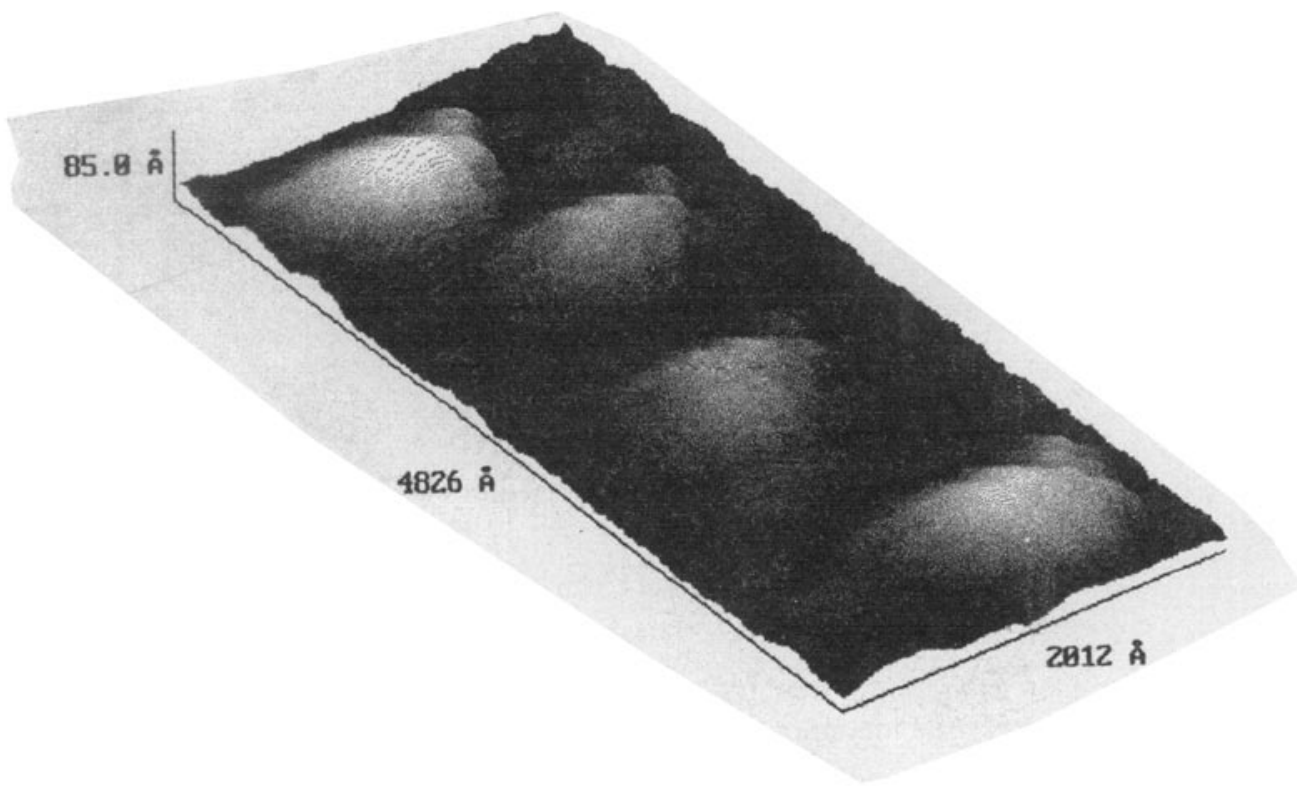

Figure 2. Nanostructures created using the same tip. The outer two were created using a voltage pulse of height $4.0 \mathrm{~V}$, whereas the inner ones are with $3.0 \mathrm{~V}$.

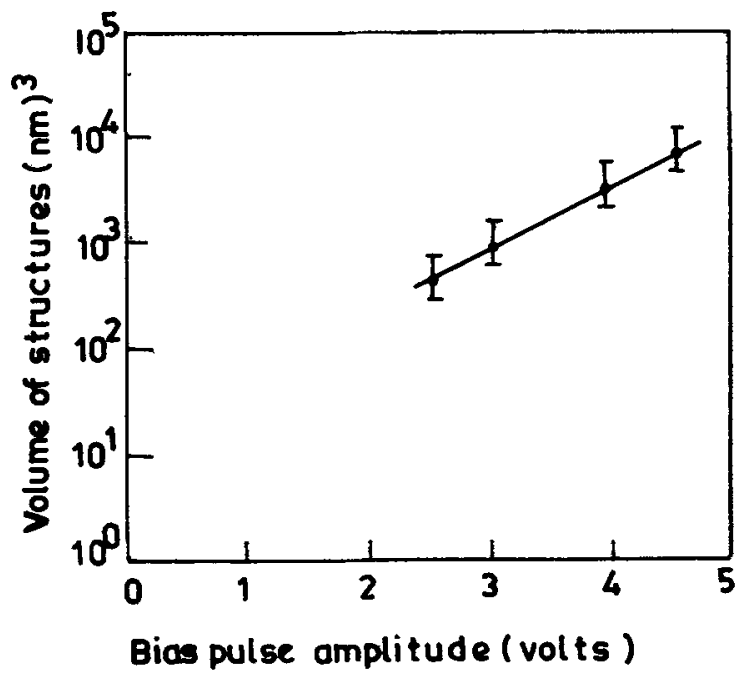

Figure 3. Characterization of nanostructures created on nickel thin film by bias pulse method. 
respect to the voltage of the bias pulse using a sharp tungsten tip. For voltage less than $2.5 \mathrm{~V}$ there is no surface modification whereas voltage above 4.5 produces drastic changes in the surface morphology. It is to be noted that the line plot may shift up or down by as much as $50 \%$ depending on the shape of the tip.

The following procedure was followed to form a single hillock using a sharp tip and a line of broad hillocks using a broad tip. After the topographic scan with constant current configuration (figure 4a) the tip was moved to the central location where the surface modification was desired. Then we switched over to the constant height mode and subsequently the voltage pulse was directly added to the tunneling voltage. The amplitude and width of the pulse recorded by digital storage oscilloscope were noted to be $4.5 \mathrm{~V}$ and $100 \mathrm{~ms}$ respectively. The transient electric field, which is created by bias pulse method, was sufficiently high to obtain plastic instead of purely elastic deformation of the surface. After the application of bias pulse the

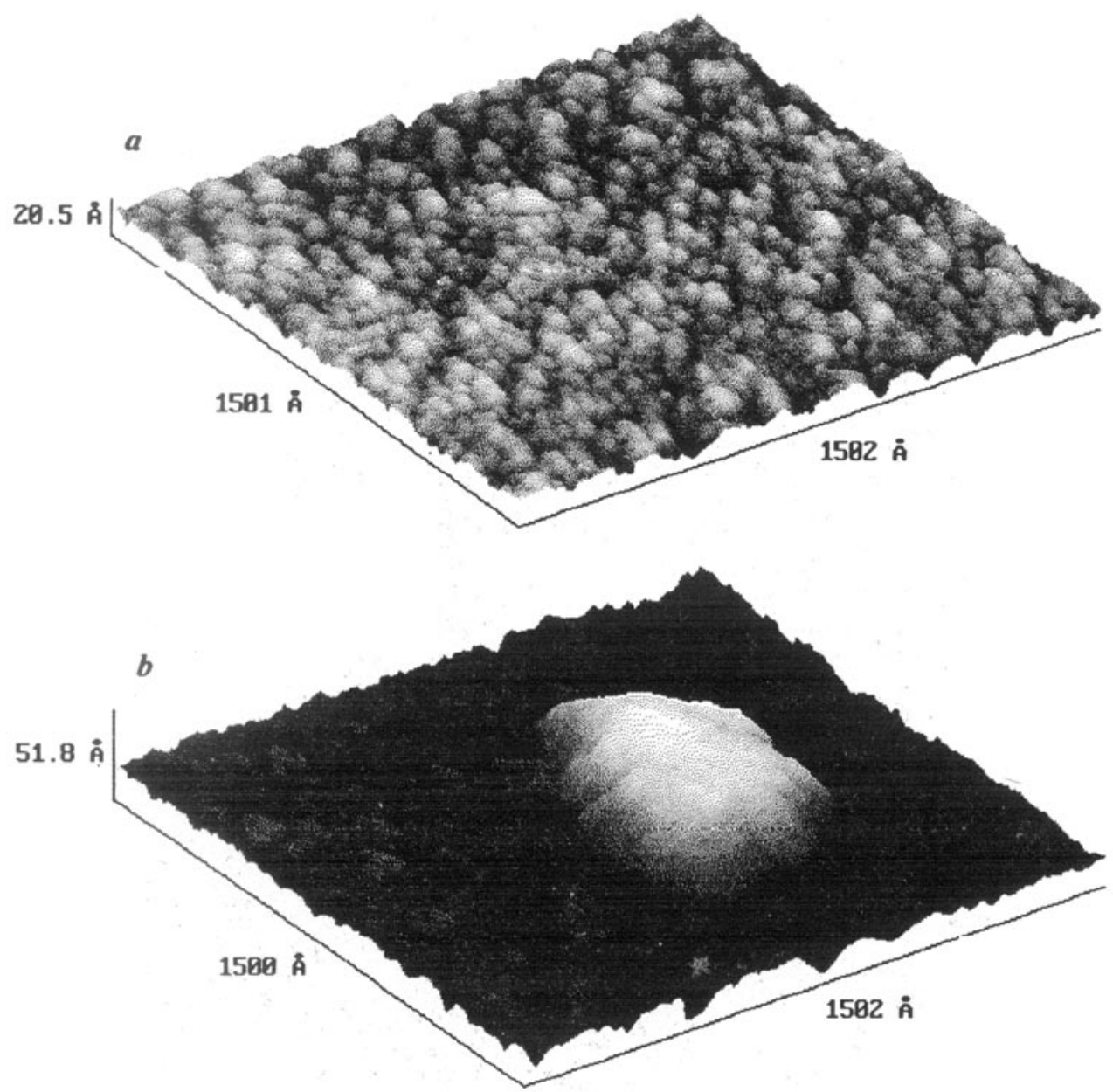

Figure 4. Formation of nanostructure by bias-pulse method (a) before and (b) after application of $2.5 \mathrm{~V}$ pulse of $100 \mathrm{~ms}$ width on nickel film. 
$a$

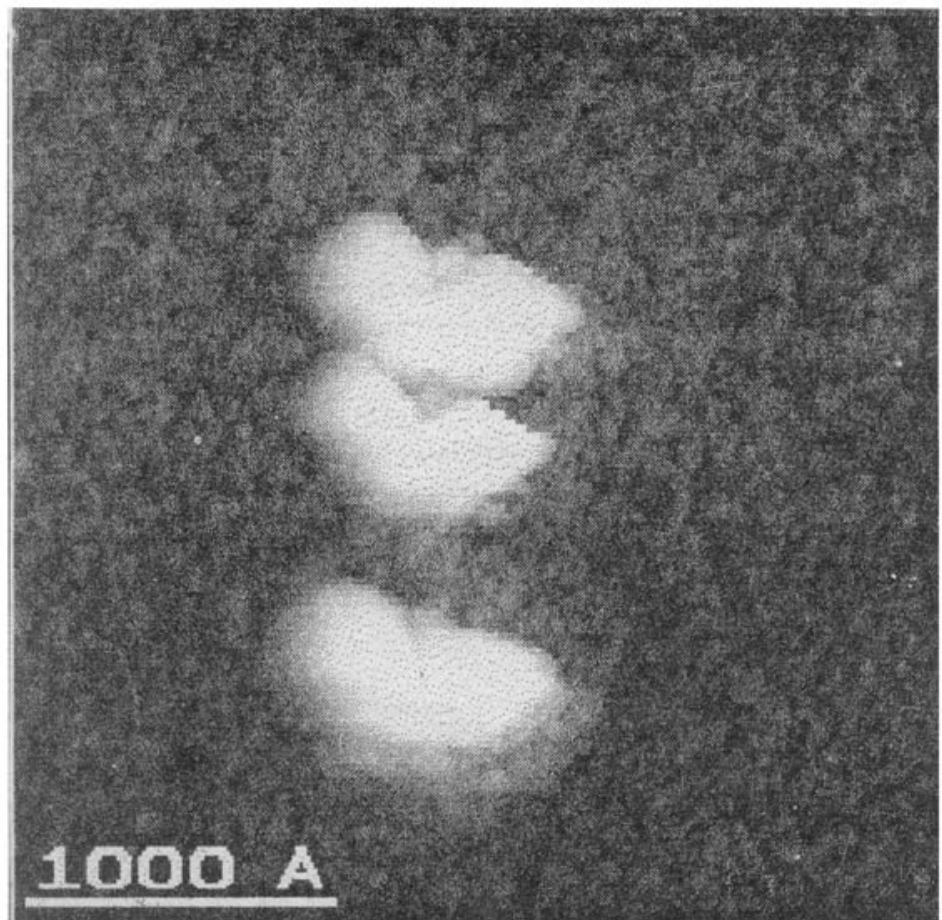

$\boldsymbol{b}$

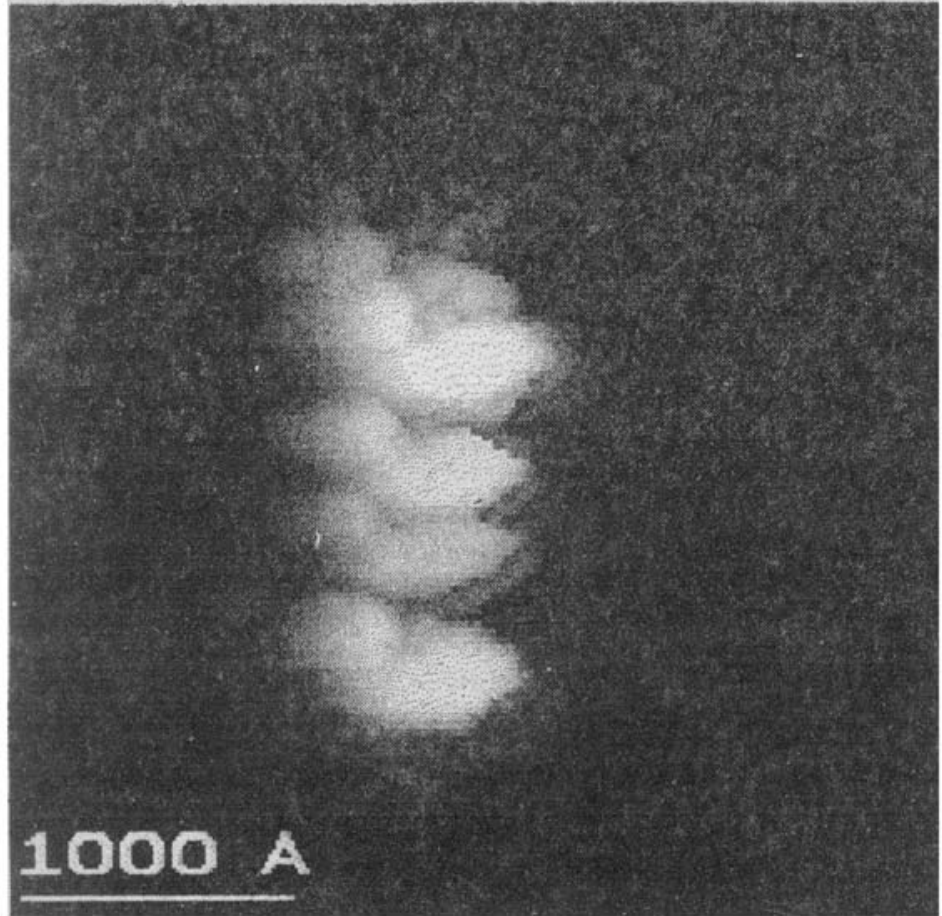

Figure 5. Creation of a line pattern by repeated application of bias pulse at different locations. Note that the discontinuity of $\mathbf{a}$ has been removed in $\mathbf{b}$ by precisely positioning the tip at the proper location. 
STM was switched back to constant current mode and the topographic image was recorded (figure $4 \mathrm{~b}$ ). It is clearly seen that a mound of $50 \mathrm{~nm}$ diameter and $5 \mathrm{~nm}$ height is created.

In order to draw a line, it is necessary to create a number of structures along the line. As the offset controls permitted us to steer the sample to bring the desired location just below the tip, we could create the line. In figure $5 \mathrm{a}$, the bias pulse signal could not reach the tip at one location, causing a discontinuity of the line. However, the structure was further modified by precisely positioning the tip in between two hillocks so as to complete the line (figure 5b). The interesting: aspect revealed by figures $5 \mathrm{a}$ and $\mathrm{b}$ is the precision of control in positioning the tip which is very crucial for writing a continuous nanostructure.

\section{Results and discussion}

The formation of hillocks is a matter of debate. When the experiments were performed on a gold surface with a gold tip (Mamin et al 1990) it was first proposed that material from the tip was getting field-evaporated and deposited on the substrate. This theory was contested by Pascual et al (1993) who argued that the formation is due to instantaneous mechanical contact between tip and sample. Tsong (1991) reviewed the various possible tip-sample atomic interactions that are possible in the field emission mode in a STM. He suggests that depending on the threshold fields for field evaporation for the tip and sample material, either of them may evaporate. In our present investigation we have used a tungsten tip for modifying nickel thin-film surfaces. We observed that even after repeated use of

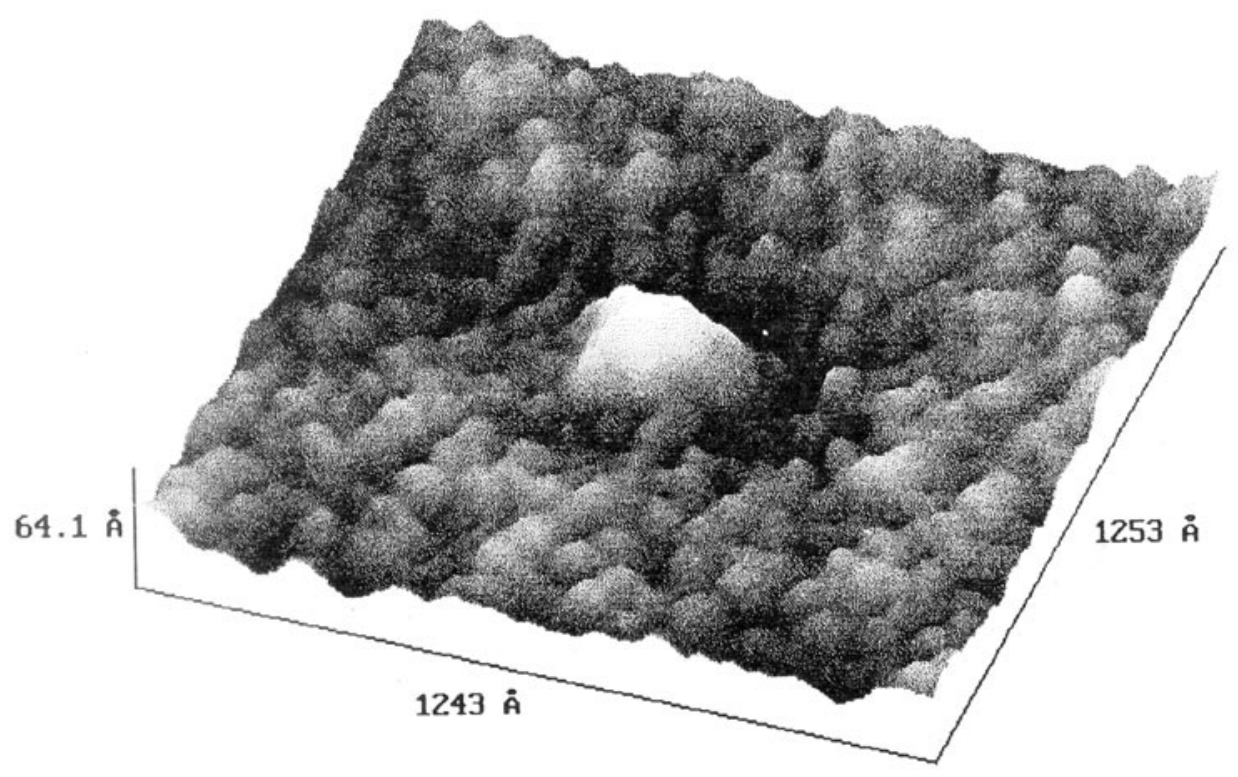

Figure 6. Formation of valley just adjacent to the hillock created by bias pulse method. 
the tip in field application mode, the quality of the STM images scanned with the same tip did not deteriorate. Secondly, as such, the movement of the tip was frozen during field application and there was no 'short circuit' between the tip and the sample and therefore the mechanical contact model (Pascual et al 1993) could not be supported. The transient electric field which is created by bias pulse method can be sufficiently high to obtain plastic instead of purely elastic deformation of the surface, in which case the mound formation can be attributed to, first, local melting and plastic deformation of film material and then its suction towards the tip by a high field gradient. The topograph in figure 6 clearly shows the existence of a valley just behind the hillock. This valley is likely to have been created during field-induced 'suction' of the surface material. Based on these observations, the 'suction' model of creation of nanostructures appears plausible.

\section{Conclusions}

Based on our observations, we conclude that nanostructures can be created on nickel thin-film surface by bias pulse technique. The structures are characterized in terms of their volume. It is found that good, reproducible structures could be created with a voltage pulse height in the range of 2.5 to $4.5 \mathrm{~V}$ and width $100 \mathrm{~ms}$. The shape of the hillock is governed by the shape of the tip but the overall size is determined by the effective field strength on the surface. The transient electric field created by bias pulse method can be sufficiently high to obtain plastic instead of purely elastic deformation of the surface, in which case the mound formation can be attributed to, first, local melting and plastic deformation of film material and then its suction towards the tip by a high field gradient.

\section{References}

Becker R S, Golovchenko J A and Swartzentruber B S 1987 Nature (London) 325419

Hartmann E, Behm R J, Krotz G, Muller G and Koch F 1991 Appl. Phys. Lett. 592136

Li Y Z, Vazquer, L, Piner R, Andreas R P and Reifnberger R 1989 A Appl. Phys. Lett. 541424

Mamin H J, Guethiner P H and Rugar D 1990 Phys. Rev. Lett. 652418

McCord, M A and Pease R F W 1986 J. Vac. Sci. Technol. B4 86

McCord M A and Pease R F W $1987 \mathrm{~J}$. Vac, Sci. Technol. B5 430

McCord M A and Pease R F.W 1988 J. Vac. Sci. Technol. B6 293

Pascual J I, Mendez J, Gomez-Herrero J, Baro A M, Garcia N and Vu Thien Binh 1993 Phys. Rev. Lett. 711852

Rao M V H and Mathur B K 1993 Indian J. Pure Appl. Phys. 31574

Rohrer H 1993 Jap. J. Appl. Phys. 321335

Saulys D, Rudd G and Garfunkel E 1991 J. Appl. Phys. 691707

Schmmel Th, Fuchs H, Akari S and Dransfeld K 1991 Appl. Phys. Lett. 581039

Schmmel Th, Eng L, Fuchs H and Lux-steiner M 1992 Ann. Chimi Fr. 17205

Schneir J, Sonnenfeld R, Marti O, Hansma P K, Demuth J E and Hamers R J 1988 J. Appl, Phys. 63717

Snow E S, Campbell P M and McMari P 1993 Appl. Phys. Lett. 63749

Staufer U, Wiesendanger R," Eng L, Rosenthaler L, Hidber H R and Guntherodt H J 1987 Appl. Phys. Lett. 51244

Staufer U, Wiesendanger R, Eng L, Rosenthaler L, Hidber H R, Guntherodt H J and Garcia N $1988 \mathrm{~J}$. Vac. Sci. Technol. A6 537

Tsong T T 1991 Phys. Rev. B44 13703

Virtanen J A, Suketu P, Huth G C and Cho Z H 1991 J. Appl. Phys. 703376 